\title{
On replica-nondiagonal large $N$ saddles in the SYK model
}

\author{
Irina Aref'eva ${ }^{1, *}, \quad$ Mikhail Khramtsov ${ }^{1, * *}, \quad$ Maria Tikhanovskaya ${ }^{1, * * *}$, and \\ Igor Volovich ${ }^{1, * * * *}$ \\ ${ }^{1}$ Steklov Mathematical Institute, Russian Academy of Sciences, \\ Gubkina str. 8, 119991, Moscow, Russia
}

\begin{abstract}
We study the saddle points of the SYK model, formulated in terms of the replica bilocal fields, beyond the replica-diagonal assumption. We find a family of replica-nondiagonal saddle points in the IR limit, where the saddle point equations are separable. We use the Parisi ansatz to find the replicanondiagonal solutions and take the replica limit. The free energy on these solutions is computed, and we find that some of the replica-nondiagonal saddles have lower free energy than the replica-diagonal saddle point.
\end{abstract}

\section{Introduction}

The object of our study is the Sachdev-Ye-Kitaev model [1-4], which is a theory of $N \gg 1$ interacting Majorana fermions in $0+1$ dimensions:

$$
H=\frac{i^{q / 2}}{q !} \sum_{i_{1}, i_{2}, \ldots, i_{q}=1}^{N} j_{i_{1} i_{2} \ldots i_{q}} \psi_{i_{1}} \psi_{i_{2}} \ldots \psi_{i_{q}} .
$$

Here $\psi_{i}$ are the Majorana fermions, and $j_{i_{1} \ldots i_{q}}$ are totally antisymmetric couplings randomized via the Gaussian distribution. To average over the disorder, one introduces replicas of the SYK. For example, the free energy is given by

$$
-\beta F=\overline{\ln Z}=\lim _{M \rightarrow 0} \frac{\ln \overline{Z^{M}}}{M} .
$$

After disorder averaging in $\overline{Z^{M}}$, one can introduce auxiliary bilocal fields $G, \Sigma$ via the Hubbard-Stratonovich transformation such that $\Sigma_{\alpha \beta}\left(\tau, \tau^{\prime}\right)$ is a Lagrange multiplier which sets $G_{\alpha \beta}\left(\tau, \tau^{\prime}\right) \sim \frac{1}{N} \sum_{i} \psi_{i}^{\alpha}(\tau) \psi_{i}^{\beta}\left(\tau^{\prime}\right)$, and $\alpha, \beta=1, \ldots, M$. After integrating out the fermions, one obtains [2-4] (in Euclidean signature):

$$
\begin{aligned}
\overline{Z(\beta)^{M}} & =\int D G D \Sigma \operatorname{Pf}\left[\delta_{\alpha \beta} \partial_{\tau}-\Sigma_{\alpha \beta}\right]^{N} \\
& \times \exp \left[-\frac{N}{2} \int_{0}^{\beta} \int_{0}^{\beta} d \tau_{1} d \tau_{2}\left(\Sigma_{\alpha \beta}\left(\tau_{1}, \tau_{2}\right) G_{\alpha \beta}\left(\tau_{1}, \tau_{2}\right)-\frac{J^{2}}{q} G_{\alpha \beta}\left(\tau_{1}, \tau_{2}\right)^{q}\right)\right],
\end{aligned}
$$

\footnotetext{
*e-mail: arefeva@mi.ras.ru

** e-mail: khramtsov@mi.ras.ru

***e-mail: tikhanovskaya@mi.ras.ru

**** e-mail: volovich@mi.ras.ru
} 
see more details about bilocal fields in SYK in [5].

The goal of the work is the analytic study of replica-nondiagonal saddle points of the disorder-averaged free energy of the SYK model. We present

- construction of solutions of the saddle point equations using simple ansatz, in which the time dependence and replica dependence are factorized.

- analysis of saddle point structure and contributions to the free energy of such solutions

The construction of the solutions is performed by employing the Parisi ansatz [6]. We solve the saddle point equations after taking the $M \rightarrow 0$ limit. The free energy is then computed by evaluating the on-shell action on the solutions.

\section{Saddle point equations and the ansatz at finite $M$}

We study the saddle points of the path integral (1) in the IR limit $\partial_{\tau} \rightarrow 0$, or, equivalently, the strong coupling regime $\beta J \gg 1$. In this case the saddle point equations read

$$
\int d \tau^{\prime} G_{\alpha \beta}\left(\tau, \tau^{\prime}\right) \Sigma_{\beta \gamma}\left(\tau^{\prime}, \tau^{\prime \prime}\right)=-\delta_{\alpha \gamma} \delta\left(\tau-\tau^{\prime \prime}\right) ; \quad \Sigma_{\alpha \beta}\left(\tau, \tau^{\prime}\right)=J^{2} G_{\alpha \beta}\left(\tau, \tau^{\prime}\right)^{q-1}
$$

We are going to study the solutions of saddle point equations using the particular ansatz, where the time and replica dependencies are factorized [7]:

$$
G_{\alpha \beta}\left(\tau, \tau^{\prime}\right)=g\left(\tau, \tau^{\prime}\right) P_{\alpha \beta}
$$

$G_{\alpha \beta}\left(\tau, \tau^{\prime}\right)=-G_{\beta \alpha}\left(\tau^{\prime}, \tau\right) \Rightarrow$ we assume that $g\left(\tau, \tau^{\prime}\right)=-g\left(\tau^{\prime}, \tau\right)$ and $P_{\alpha \beta}=P_{\beta \alpha}$. Substituting second equation into the first one in (2), using the ansatz (3) and taking the diagonal component with $\alpha=\gamma$ we get the equation

$$
J^{2} \mathcal{C} \int d \tau^{\prime} g\left(\tau, \tau^{\prime}\right) g\left(\tau^{\prime}, \tau^{\prime \prime}\right)^{q-1}=-\delta\left(\tau-\tau^{\prime \prime}\right),
$$

The constant $\mathcal{C}$ is defined by the matrix $P$ as $\sum_{\beta} P_{\alpha \beta}^{q}=\mathcal{C}, \quad \forall \alpha$. Together with the off-diagonal component of the equation (2) on the factorized ansatz, this yields the equation of motion for the matrix $P$ :

$$
\sum_{\beta} P_{\alpha \beta} P_{\beta \gamma}^{q-1}=\delta_{\alpha \gamma} \mathcal{C}
$$

The appearance of the new apparent free parameter $\mathcal{C}$ is caused by the fact that the ansatz (3) allows for a scaling ambiguity. In this work we fix the scaling in such a way that $P_{\alpha \alpha}=1$.

Equation (4) up to normalization is the same equation as the equation for the replicasymmetric solution, so we can readily write down the solution (at finite temperature):

$$
g\left(\tau, \tau^{\prime}\right)=\frac{1}{\mathcal{C}^{1 / q}} g_{c}\left(\tau-\tau^{\prime}\right)
$$

where at finite temperature

$$
g_{c}(\tau)=b\left(\frac{\pi}{\beta}\right)^{2 / q} \frac{\operatorname{sgn}(\tau)}{\left|\sin \frac{\pi}{\beta} \tau\right|^{2 / q}}, \quad \text { where } \quad b^{q}=\frac{(q-2) \tan \frac{\pi}{q}}{2 \pi q J^{2}}
$$

In the frequency space the conformal propagator on a circle has a form [8]:

$$
g_{c}\left(\omega_{n}\right)=-i 2 b\left(\frac{2 \pi}{\beta}\right)^{2 / q-1} \cos (\pi / q) \frac{\Gamma\left(\frac{\beta}{2 \pi} \omega_{n}+\frac{1}{q}\right) \Gamma\left(1-\frac{2}{q}\right)}{\Gamma\left(\frac{\beta}{2 \pi} \omega_{n}+1-\frac{1}{q}\right)},
$$


where $\omega_{n}$ are Matsubara frequencies $\omega_{n}=\frac{2 \pi}{\beta}\left(n+\frac{1}{2}\right), n \in \mathbb{Z}$. At zero temperature (7) and (8) reduce to

$$
g_{c}(\tau)=\frac{b}{|\tau|^{2 / q}} \operatorname{sgn}(\tau), \quad g_{c}(\omega)=i b 2^{1-\frac{2}{q}} \sqrt{\pi} \frac{\Gamma\left(1-\frac{1}{q}\right)}{\Gamma\left(\frac{1}{2}+\frac{1}{q}\right)}|\omega|^{\frac{2}{q}-1} \operatorname{sgn}(\omega),
$$

In order to solve the equations (5) we assume that $P$ has the Parisi matrix form. Let us remind the definition of the Parisi matrices.

We define the Parisi matrix $P$ in terms of the Parisi algebra generators $\mathcal{I}_{m_{i}}$, that have the form $\mathcal{I}_{m_{i}}=I_{M / m_{i}} \otimes \mathfrak{J}_{m_{i}}$, where $\left(\mathfrak{J}_{i}\right)_{k j}=1, k, j=1, \ldots, m_{i}$. Here $I_{p}$ is a unit matrix of dimension $p$. An arbitrary Parisi matrix $P$ is expanded in terms of the generators as

$$
P=\sum_{i=1, m_{i} \in \mathfrak{I}}^{l} a_{i}\left(\mathcal{I}_{m_{i+1}}-\mathcal{I}_{m_{i}}\right)+a_{0} \mathcal{I}_{1}
$$

Using the algebra $\mathcal{I}_{m_{i}} \mathcal{I}_{m_{j}}=\mathcal{I}_{m_{j}} \mathcal{I}_{m_{i}}=m_{i} \mathcal{I}_{m_{j}}$, for $i \leq j$ we can calculate a product of two Parisi matrices which participate in the equation (5) (in the calculations we will fix $P_{\alpha \alpha}=a_{0}=1$ ):

$$
P=\sum_{i=1, m_{i} \in \mathfrak{I}}^{l} a_{i}\left(\mathcal{I}_{m_{i+1}}-\mathcal{I}_{m_{i}}\right)+\mathcal{I}_{1}, \quad P^{\circ(q-1)}=\sum_{i=1, m_{i} \in \mathfrak{I}}^{l} a_{i}^{q-1}\left(\mathcal{I}_{m_{i+1}}-\mathcal{I}_{m_{i}}\right)+\mathcal{I}_{1} .
$$

Here $\circ$ is the Hadamard multiplication. Taking the diagonal and off-diagonal components of the resulting Parisi matrix, we obtain the equation (5) in terms of Parisi variables:

$$
\begin{gathered}
\mathcal{C}=1+\sum_{j=1}^{l} a_{j}^{q}\left(m_{j+1}-m_{j}\right) \\
0=a_{j}+a_{j}^{q-1}+\sum_{i<j}\left(a_{i} a_{j}^{q-1}+a_{j} a_{i}^{q-1}\right)\left(m_{i+1}-m_{i}\right)-m_{j} a_{j}^{q}+\sum_{i>j-1} a_{i}^{q}\left(m_{i+1}-m_{i}\right) .
\end{gathered}
$$

\section{On-shell action on factorized solutions}

We start with the on-shell action for the partition function (1) at finite $M$ in the strong coupling limit,

$$
\frac{2}{N} S_{M}=-\operatorname{Tr} \log \left(-\Sigma_{\alpha \beta}\right)+\left.\int_{0}^{\beta} \int_{0}^{\beta} d \tau_{1} d \tau_{2} \sum_{\alpha, \beta}\left(G_{\alpha \beta}\left(\tau_{1}, \tau_{2}\right) \Sigma_{\alpha \beta}\left(\tau_{1}, \tau_{2}\right)-\frac{J^{2}}{q} G_{\alpha \beta}\left(\tau_{1}, \tau_{2}\right)^{q}\right)\right|_{\text {on-shell }} .
$$

To compute the on-shell action, we need to evaluate the Pfaffian. Performing the integration over Majorana fermions in the frequency space, we can show that it factorizes on the ansatz (3):

$$
\begin{aligned}
\operatorname{Pf}\left(-\Sigma_{\alpha \beta}\right) & =\int \prod_{0<n<d_{f}} \prod_{\alpha} d \tilde{\chi}_{\alpha}\left(\omega_{n}\right) d \overline{\tilde{\chi}}_{\alpha}\left(\omega_{n}\right) \exp \left(-\frac{1}{\beta} \sum_{0<n<d_{f}} \overline{\tilde{\chi}}_{\alpha}\left(\omega_{n}\right) \tilde{\Sigma}_{c}\left(\omega_{n}\right) \mathcal{C}^{\Delta-1} P_{\alpha \beta}^{q-1} \tilde{\chi}_{\beta}\left(\omega_{n}\right)\right) \\
& =\prod_{0<n<d_{f}}\left(-\frac{1}{\beta} \tilde{\Sigma}_{c}\left(\omega_{n}\right)\right)^{M / 2} \times \operatorname{det}\left(\mathcal{C}^{\Delta-1} P_{\alpha \beta}^{q-1}\right)^{d_{f} / 2},
\end{aligned}
$$


where we use the saddle point equations (2) and substitute the factorized ansatz (3). We obtain the resulting expression for the on-shell action in the form $\frac{2}{N} S_{M}=M\left(\mathfrak{s}_{1, c}+\mathfrak{s}_{2, c}+\mathfrak{s}_{3}\right)$, where

$$
\begin{aligned}
\mathfrak{s}_{1, c} & =-\sum_{0<n<d_{f}} \log \left(-\frac{1}{\beta} \tilde{\Sigma}_{c}\left(\omega_{n}\right)\right) ; \quad \mathfrak{s}_{2, c}=d_{f}\left(1-\frac{1}{q}\right) J^{2} ; \\
\mathfrak{s}_{3} & =-\frac{d_{f}}{M} \log \operatorname{det}\left[\mathcal{C}^{1 / q-1} P^{\circ(q-1)}\right],
\end{aligned}
$$

and on the saddle point $\overline{Z^{M}} \sim \mathrm{e}^{-S_{M}}$. Note that we work in the leading order of the strong coupling limit. The terms $\mathfrak{s}_{1, c}$ and $\mathfrak{s}_{2, c}$ are the same as in the replica-diagonal solution, but the replica matrix $P$ gives a new contribution to the on-shell action $\mathfrak{s}_{3}$, which is what we will focus on. We impose hard cutoff $d_{f}$ on Matsubara frequencies in such a way that the IR limit is valid: $\left|\omega_{n}\right| \leq J$, which means we have to set $d_{f} \sim \beta J$.

\section{The $M \rightarrow 0$ limit}

The replica trick requires the analytic continuation and taking the limit $M \rightarrow 0$ in the saddle point equation (5) and on-shell action. We analytically continue from the discrete set of components of a Parisi matrix to the Parisi function $a_{j} \rightarrow a(u)$. After taking $M \rightarrow 0$ in the equations (12), defining the average $\int_{0}^{1} a^{p}(v) d v \equiv\left\langle a^{p}\right\rangle$, we arrive at the following equations:

$$
\begin{gathered}
\mathcal{C}=1-\left\langle a^{q}\right\rangle \\
0=a(u)\left[1-\left\langle a^{q-1}\right\rangle\right]+a^{q-1}(u)[1-\langle a\rangle]-\int_{0}^{u}[a(v)-a(u)]\left[a^{q-1}(v)-a^{q-1}(u)\right] d v,
\end{gathered}
$$

where $u \in[0,1]$. Defining the Parisi matrix $Q=\mathcal{C}^{1 / q-1} P^{\circ(q-1)}$, the contribution to the free energy is reads $\Delta F=F_{R N D}-F_{R D}$ (here RD and RND means replica diagonal and replica non-diagonal cases, respectively) and

$$
\Delta F=\left.\lim _{M \rightarrow 0} \frac{N}{2 \beta} \mathfrak{s}_{3}\right|_{\text {reg }}=-\lim _{M \rightarrow 0} \frac{N}{2 \beta} \frac{1}{M} d_{f} \operatorname{tr} \log Q=-\lim _{M \rightarrow 0} \frac{J N}{2} \frac{1}{M} \operatorname{tr} \log Q .
$$

The tracelog is computed using the Parisi formula:

$$
\begin{aligned}
& -\lim _{M \rightarrow 0} \frac{1}{M} \operatorname{tr} \log Q=-\log \left(q_{0}-\langle q\rangle\right)-\frac{q(0)}{q_{0}-\langle q\rangle}+\int_{0}^{1} \frac{d v}{v^{2}} \log \frac{q_{0}-\langle q\rangle-[q](v)}{q_{0}-\langle q\rangle} \\
= & -\frac{1-q}{q} \log \mathcal{C}-\log \left(1-\left\langle a^{q-1}\right\rangle\right)-\frac{a^{q-1}(0)}{1-\left\langle a^{q-1}\right\rangle}+\int_{0}^{1} \frac{d v}{v^{2}} \log \frac{1-\left\langle a^{q-1}\right\rangle-\left[a^{q-1}\right](v)}{1-\left\langle a^{q-1}\right\rangle} .
\end{aligned}
$$

We note that, as we will demonstrate, we can have complex saddles. In this case, we take the real part in (18), because the contribution from imaginary part vanishes in the large $N$ limit [9]. The complex saddles also do contribute in the zero-dimensional SYK model [11].

\section{Solutions}

\subsection{Replica symmetric solutions}

We start the discussion of the solutions of equations for $P$ with the simplest replicanondiagonal case, the replica-symmetric solutions. Let us take $P_{\alpha \alpha}=1, \alpha=1,2, \ldots, M$ and 
$P_{\alpha \beta}=A$ if $\alpha \neq \beta$. Thus this family of solutions is parametrized by a single $c$-number $A$. We have

$$
P=\left(P_{\alpha \beta}\right)=\left(\begin{array}{cccc}
1 & A & A & \ldots \\
A & 1 & A & \ldots \\
A & A & 1 & \ldots \\
. & . & . & \ldots
\end{array}\right), P^{\circ(q-1)}=\left(\begin{array}{cccc}
1 & A^{q-1} & A^{q-1} & \ldots \\
A^{q-1} & 1 & A^{q-1} & \ldots \\
A^{q-1} & A^{q-1} & 1 & \ldots \\
. & . & . & \ldots
\end{array}\right)
$$

Note that

$$
\operatorname{det}\left(P^{\circ(q-1)}\right)=\left(1-A^{q-1}\right)^{M-1}\left(1+(M-1) A^{q-1}\right)
$$

and one has

$$
\lim _{M \rightarrow 0} \frac{1}{M} \log \operatorname{det}\left(P^{\circ(q-1)}\right)=\log \left(1-A^{q-1}\right)+\frac{A^{q-1}}{1-A^{q-1}}
$$

Equation (5) yields

$$
A^{q-1}+A+A^{q}(M-2)=0, \quad \mathcal{C}=1+A^{q}(M-1)
$$

The limit $M \rightarrow 0$ is readily taken in this case, and the equations take the form

$$
A^{q-1}+A-2 A^{q}=0, \quad \mathcal{C}=1-A^{q} .
$$

We aim to compute the limit of (18) at $M \rightarrow 0$. We have

$$
-\lim _{M \rightarrow 0} \frac{1}{M} \log \operatorname{det}\left(C^{\frac{1}{q}-1} P^{\circ(q-1)}\right)=\frac{q-1}{q} \log \left(1-A^{q}\right)-\log \left(1-A^{q-1}\right)-\frac{A^{q-1}}{1-A^{q-1}}
$$

Equation (24) has a pair of two complex conjugated roots $A=b, A=\bar{b}$. Equation for $q=2(n-1)$ has $n$ pairs of conjugated roots. One pair of saddle points gives contribution to the difference $\Delta F=F_{D N D}-F_{R D}$ between replica diagonal and replica non-diagonal cases

$$
f_{q} \equiv \frac{2}{N J} \Delta F_{q}=\left(\frac{q-1}{q} \log \left|1-b^{q}\right|-\log \left|1-b^{q-1}\right|-\operatorname{Re} \frac{b^{q-1}}{1-b^{q-1}}\right)
$$

Below we present pairs of nontrivial solutions $A=\left(b_{q}^{(i)}, \bar{b}_{q}^{(i)}\right), i=1, \ldots \frac{q}{2}-1$ to (24) for $q=4,6,8,10$ and the corresponding free energy $f_{q}$ :

\begin{tabular}{|l|l|l|}
\hline $\mathrm{q}$ & $b_{q}$ & $f_{q}$ \\
\hline 4 & $b_{4}^{(1)}=(-1 \pm i \sqrt{7}) / 4$ & $f_{4}^{(1)}=-0.028$ \\
\hline \multirow{2}{*}{6} & $b_{6}^{(1)}=0.621 \pm 0.502 i ;$ & $f_{6}^{(1)}=0.0652 ;$ \\
& $b_{6}^{(2)}=0.371-0.803 i$ & $f_{6}^{(2)}=-0.301$ \\
\hline \multirow{4}{*}{8} & $b_{8}^{(1)}=-0.757 \pm 0.388 i ;$ & $f_{8}^{(1)}=0.104 ;$ \\
& $b_{8}^{(2)}=-0.137 \pm 0.869 i ;$ & $f_{8}^{(2)}=-0.080 ;$ \\
& $b_{8}^{(3)}=0.644 \pm 0.690 i$ & $f_{8}^{(3)}=-0.556$ \\
\hline \multirow{5}{*}{10} & $b_{10}^{(1)}=-0.824 \pm 0.314 i ;$ & $f_{10}^{(1)}=0.125 ;$ \\
& $b_{10}^{(2)}=-0.415 \pm 0.79 i ;$ & $f_{10}^{(2)}=0.0132 ;$ \\
& $b_{10}^{(3)}=0.215 \pm 0.898 i ;$ & $f_{10}^{(3)}=-0.244 ;$ \\
& $b_{10}^{(4)}=0.775 \pm 0.582 i$ & $f_{10}^{(4)}=-0.773$ \\
\hline
\end{tabular}

Note, that the contribution to the free energy $f_{4}$ on pair $\left(b_{4}^{(1)}, \bar{b}_{4}^{(1)}\right)$ admit the analytical expression $f_{4}^{(1)}=-\frac{3}{8}+\frac{\log 2}{2}=-0.028$ and it is below the replica diagonal case. All other contributions are plotted on Fig.1A. We see negative values of $f_{q}$ for $q>4$. The dependence of the free energy of the lowest replica-symmetric saddle on $q$ is presented on Fig.1B. 

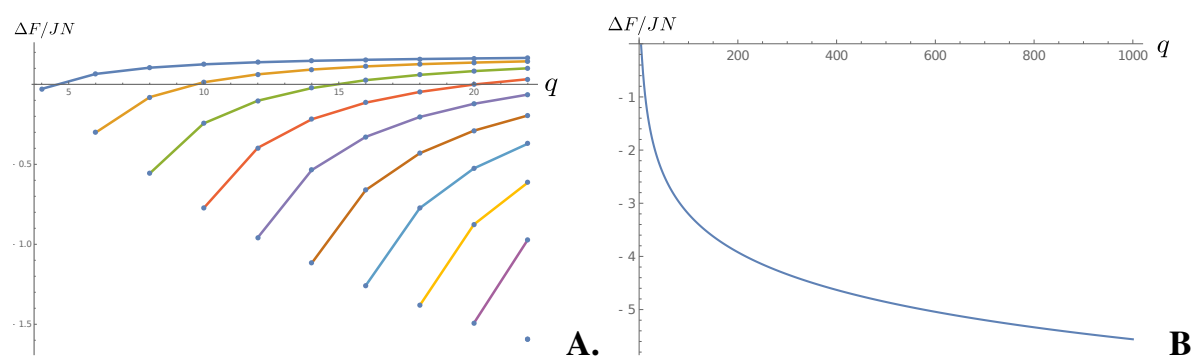

B.

Figure 1: A. Free energy on replica-symmetric saddles at various values of $q$. B. Free energy of the lowest replica-symmetric saddle as a function of $q$.

\subsection{One-step replica symmetry breaking solutions}

We restrict ourselves to the solutions for $a(u)$, which can be described by the one-step replica symmetry breaking ansatz, in analogy with the spin glass systems [6], such as the Sachdev-Ye model [10] (we fix $q=4$ ):

$$
a(u)=A_{0}+A_{1} \theta(u-\mu) .
$$

In this formula $\mu$ is a free parameter, to which we refer as the breakpoint, in analogy with the spin glass solutions [10]. The special cases of this ansatz reproduce the replica-symmetric solutions in the following manner:

- Replica-diagonal (paramagnetic) solution: $A_{0}=0, A_{1}=0$.

- Replica-symmetric solution with $A_{0}=1, A_{1}=0$. This solution does not describe a saddle of the path integral (1), because it has $\mathcal{C}=0$.

- Replica-symmetric complex-valued solutions, discussed above. In the $q=4$ case they are shown by the pair of green points on Fig.3A.

Besides these solutions, we also have 12 solutions with replica symmetry breaking, i.e. nnzero $A_{1}$ :

- There are one real and two complex solutions with $A_{0}=0$. The expressions for $A_{1}$ can be found analytically:

$$
\begin{aligned}
A_{1}^{(1)} & =\frac{\sqrt[3]{2}}{3(2-\mu) \mathfrak{K}}+\frac{\Re}{3 \sqrt[3]{2}(2-\mu)}+\frac{1}{3(2-\mu)} \\
A_{1}^{(2,3)} & =-\frac{(1 \mp i \sqrt{3})}{32^{2 / 3}(2-\mu) \Re}-\frac{(1 \pm i \sqrt{3}) \Re}{6 \sqrt[3]{2}(2-\mu)}+\frac{1}{3(2-\mu)},
\end{aligned}
$$

where

$$
\Re=\sqrt[3]{-\mathfrak{f}-\sqrt{\mathfrak{f}^{2}-4}}, \quad \mathfrak{f}=-27 \mu^{2}+108 \mu-110 .
$$

The location of solution (29) is shown on Fig.2A, and the behavior of its contribution to the free energy as a function of $\mu$ is presented on Fig.2B. We see that the minimal value of the free energy on this family of solutions corresponds is achieved at some critical value of the breakpoint parameter $\mu \in(0,1)$, and the value of the free energy is lower than $F_{R D}$. On the other hand, we find that for the real solution (28) the minimum is achieved on the replica-diagonal value at $\mu=1$. 
- Second group of RSB solutions is characterized by non-zero both $A_{0}$ and $A_{1}$. Locations of these saddle points are presented on Fig. 3 and free energy contributions are presented on Fig. 4 by the corresponding colors. We see that the cyan, blue and dark red solutions achieve minimum of the free energy at some values of $\mu \in(0,1)$, and in those points $\Delta F<0$.
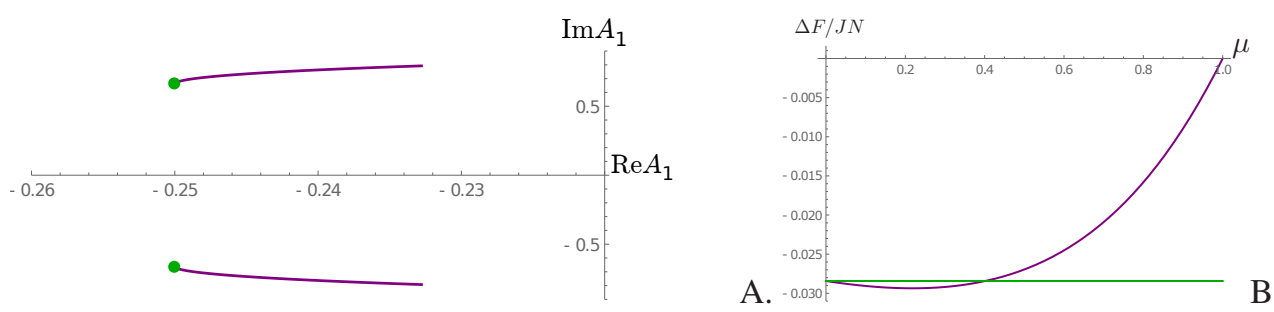

Figure 2: A. Trajectories of complex saddle points with $A_{0}=0$. B. The value of the free energy on these solutions. The green points show the location of replica-symmetric solution and the corresponding real part of the free energy density. Here $q=4$.

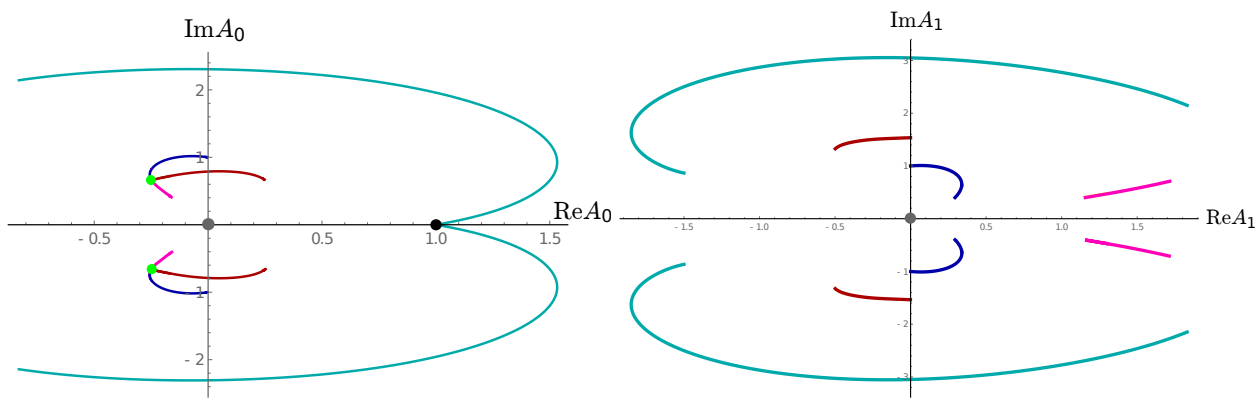

Figure 3: Trajectories of saddle points with non-zero $A_{0}$ and $A_{1}$, parametrized by $\mu$ on the complex plane. Here $q=4$.

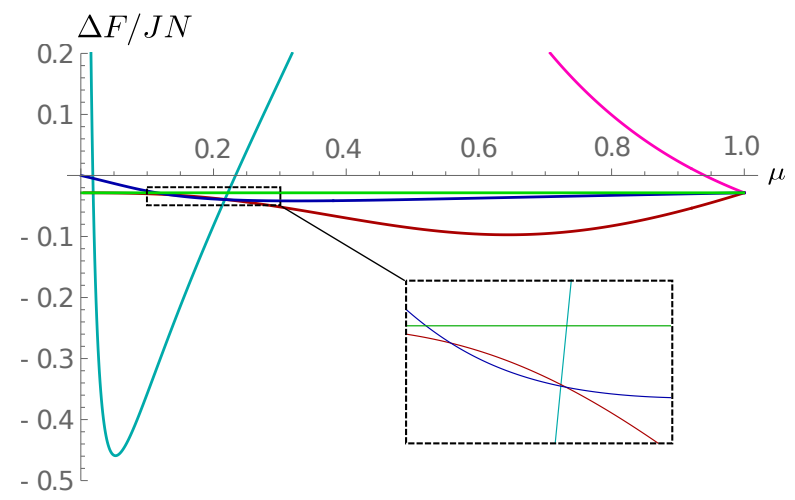

Figure 4: Free energy density on the solutions as function of $\mu$. Here $q=4$. 


\section{Conclusions and open questions}

- We analytically constructed a family of replica-nondiagonal solutions using the Parisi ansatz in SYK. It was done by solving the saddle point equations in the conformal limit after taking the limit $M \rightarrow 0$. The resulting integral equation (17) is turned into an algebraic when using the one-step RSB ansatz.

- The replica structure of the solutions contributes to the free energy. There are replicanondiagonal saddles in both replica-symmetric and RSB families with free energy lower than the replica-diagonal value.

We have two main questions that remain to be answered in the future work. The first issue is the question of the UV-completion of the factorized saddles away from the IR regime and consistency of the adopted regularization approach in the free energy. The second question is the stability of the discussed saddle points with respect to the replica-nondiagonal fluctuations. We expect that our findings regarding the non-trivial saddle points of the SYK model at strong coupling might be useful to gain new perspective into $2 \mathrm{~d}$ quantum gravity via holography.

\section{Acknowledgments}

This contribution to the Quarks 20018 proceedings is based on the paper [9] and was presented by M. T. at the Conference. I. A., M. K. and M. T. are grateful to the organizers of the Quarks 2018 conference for the opportunities to give a talk and for inspiring atmosphere during the conference. M. K. and M. T. are supported by the RFBR grant 18-32-00918.

\section{References}

[1] S. Sachdev and J. Ye, Phys. Rev. Lett. 70, 3339 (1993)

[2] A. Kitaev, talks at KITP in 2015: http://online.kitp.ucsb.edu/online/entangled15/kitaev/, http://online.kitp.ucsb.edu/online/entangled15/kitaev2/

[3] J. Maldacena and D. Stanford, Phys. Rev. D 94, no. 10, 106002 (2016)

[4] A. Kitaev and S. J. Suh, JHEP 1805, 183 (2018)

[5] I. Aref'eva, M. Khramtsov, M. Tikhanovskaya, On 1/N diagrammatics in the SYK model beyond the conformal limit", Quarks 2018 proceedings.

[6] M. Mezard and G. Parisi, "Replica field theory for random manifolds," LPTENS-90-28.

[7] A. Kamenev, talk at Steklov Mathematical Institute, March 2018

[8] R. Gurau, "The $\imath \epsilon$ prescription in the SYK model," arXiv:1705.08581 [hep-th].

[9] I. Aref'eva, M. Khramtsov, M. Tikhanovskaya and I. Volovich, "Replica-nondiagonal solutions in the SYK model," in preparation.

[10] A. Georges, O. Parcollet and S. Sachdev, Phys. Rev. B 63 (2001) 134406

[11] I. Aref 'eva and I. Volovich, "Notes on the SYK model in real time," Theoret. and Math. Phys., vol.196 (2018), arXiv:1801.08118 [hep-th]. 